\title{
General existence of competitive equilibrium in the growth model with an endogenous labor-leisure choice*
}

\author{
Aditya Goenka $\quad$ Manh-Hung Nguyen ${ }^{\ddagger}$
}

September 2, 2020

\begin{abstract}
We prove the existence of competitive equilibrium in the canonical optimal growth model with elastic labor supply under general conditions. In this model, strong conditions to rule out corner solutions are often not well justified. We show using a separation argument that there exist Lagrange multipliers that can be viewed as a system of competitive prices. Neither Inada conditions, nor strict concavity, nor homogeneity, nor differentiability are required for existence of a competitive equilibrium. Thus, we cover important specifications used in the macroeconomics literature for which existence of a competitive equilibrium is not well understood. We give examples to illustrate the violation of the conditions used in earlier existence results but where a competitive equilibrium can be shown to exist following the approach in this paper.
\end{abstract}

Keywords: Optimal growth; Competitive equilibrium; Lagrange multipliers; Elastic labor supply; Inada conditions.

JEL Classification: C61, D51, E13, 041

\footnotetext{
${ }^{*}$ Corresponding author: Manh-Hung Nguyen. We would like to thank Professors Atsushi Kajii, M. Ali Khan, Cuong Le Van and two anonymous referees for helpful suggestions and comments. Manh-Hung Nguyen acknowledges support from ANR under grant ANR-17-EURE-0010 (Investissements d'Avenir program)

${ }^{\dagger}$ Department of Economics, University of Birmingham, Birmingham B15 2TY. Email: a.goenka@bham.ac.uk

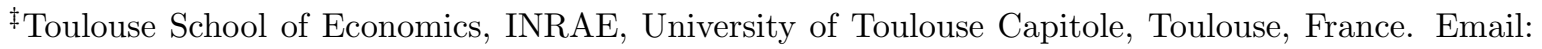
manh-hung.nguyen@tse-fr.eu.
} 


\section{Introduction}

The optimal growth model is one of the main frameworks in macroeconomics. While variations of the model with inelastic labor supply are used widely in growth theory, the version with elastic labor supply is the canonical model in business cycle models, both for exogenous and endogenous fluctuations. ${ }^{1}$ Despite the central place of the model in dynamic general equilibrium models, existence of competitive equilibrium in general settings has proved to be a challenge. Results of existence of equilibrium for this model use strong conditions (see Coleman (1997), Datta, et al. (2002), Greenwood and Huffman (1995), Le Van and Vailakis (2004), and Yano (1989, 1990, 1998)) which are often violated in models of special interest. This paper establishes existence of equilibrium under very weak conditions: neither Inada conditions, nor strict concavity, nor differentiability, nor constant returns to scale (or more generally, homogeneity), nor restrictions on cross-partials of the utility functions, nor interiority assumptions. The recent paper by Kamihigashi (2015) shows that even if we make all the above assumptions (but not Inada or stronger assumptions) then there may be no interior optimal paths. ${ }^{2}$ Understanding existence of both optimal and competitive equilibria in this model when we may not have interior paths still remains an open issue. Our results show that existence of both optimal and competitive paths can be established under very weak conditions, and whether the path is interior or not, is not important.

The approach taken in this paper is a direct method based on existence of Lagrange multipliers to the optimal problem and their representation as a summable sequence. The price of the good is the multiplier on the resource constraint. Thus, we not only know there exist equilibrium prices, but we can also calculate them in a given model. This is important as we would like to be able to characterize the equilibrium prices especially when we have non-interior equilibrium paths where existing methods do not apply. We give three examples where we can calculate equilibrium prices where the results in the literature are inapplicable.

The problem with inelastic labor supply was considered by Le Van and Saglam (2004). This approach uses a separation argument where the multipliers are represented in the dual space $\left(\ell^{\infty}\right)^{\prime}$ of the space of bounded sequences $\ell^{\infty}{ }^{3}$ The Le Van and Saglam (2004) approach uses a separation argument but imposes restrictions on the asymptotic behavior

\footnotetext{
${ }^{1}$ See the recent papers Iwasa and Sorger (2018) and Sorger (2018).

${ }^{2}$ The cited paper does not study competitive equilibria.

${ }^{3}$ While one would like the multipliers and prices to lie in $\ell^{1}$, it is not the dual space. In the previous work on competitive equilibrium following Peleg and Yaari (1970), the representation theorems followed separation arguments applied to arbitrary vector spaces (See Bewley (1972), Aliprantis, et al. (1997), Dana and Le Van (1991)).
} 
of the objective functional and constraint functions which are easily shown to be satisfied in standard models. ${ }^{4}$ There is a difficulty in going from the inelastic labor supply to the elastic labor supply model: While one may be able to show that the optimal capital stock is strictly positive, one cannot be sure that the optimal labor supply sequence is strictly positive. Thus, the paper by Le Van and Vailakis (2004) which took the approach of decentralizing the optimal solution via prices as marginal utilities had to make additional strong conditions on the utility function (which fails in homogeneous utility functions such as those of the Cobb-Douglas class) to ensure that the labor supply sequence remains strictly positive. We extend Le Van and Saglam (2004) and show the Lagrange multipliers to the social planners problem are a summable sequence and one can directly use these to decentralize the optimal solution without having to make strong assumptions to ensure interiority of the optimal plan. ${ }^{5}$ As the separation theorem does not require strict concavity or differentiability, these strong assumptions on utility functions can be dropped. This is of interest as an important specification of preferences in applied macroeconomics models are quasi-linear utility with linear utility of leisure where strict concavity and Inada conditions are violated. The linear specification also results in the planners problem in models with indivisible labor (see Hansen (1985), and Rogerson (1988)). Furthermore, for CES functions, Inada condition can be violated. In calibrated models the competitive equilibria essentially result in an interior solution but the the problem is more fundamental: While for some examples we can calculate the equilibrium allocation, we still have to show that there always exist equilibrium prices that are summable. We give the main result on existence of a competitive equilibrium by showing that the price sequence constructed is an equilibrium one. Furthermore, there is no need to make any assumption on cross-partial derivatives of the utility function. ${ }^{6}$ Thus, as one would expect, whether labor supply is backward bending or not, and whether consumption is interior or not plays no role in existence of equilibrium. As only convexity and not differentiability is required for the separation theorem we are also able to cover Leontief and more generally linear activity analysis models that are not covered by the existing results.

\footnotetext{
${ }^{4}$ This is related to Dechert (1982).

${ }^{5}$ Goenka, et al. (2012) in a model with heterogeneous agents also assume Inada conditions. While there is an interior solution for aggregate variables, the consumption and leisure of the more impatient consumers converge to zero as time tends to infinity.

${ }^{6}$ See Aiyagari, et al. (1992), Coleman (1997), Datta, et al. (2002), Greenwood and Huffman (1995), and Le Van, et al. (2007). These papers essentially show the isomorphism of the dynamic problem with endogenous leisure to one without endogenous leisure, and the assumptions are used to show monotonicity of the optimal capital path which combined with the static labor-leisure choice gives existence in the original problem. Making these assumptions rules out non-linear dynamics and thus, is overly restrictive (see Iwasa and Sorger (2018) and Sorger (2018)).
} 
Yano $(1984,1990,1998)$ also studies existence of competitive equilibrium with endogenous labor under general conditions. There are both produced input/consumption goods (i.e. capital) and non-produced input/consumption goods (which can be interpreted as labor/leisure). While the conditions in these papers weaken the conditions used in Bewley (1982) they do not cover our existence result. Yano (1984) has the most general specification and is the closest to our assumptions. It does not use differentiability (and hence, Inada conditions). It also does not use interiority assumptions in Bewley (1982). However, it makes assumptions A.14-A.17 that we do not have to make. In our results as we are concerned only with the existence issue we allow for corner solutions. In particular, we give an example (Example 3) where the consumption of the produced good is zero (except in the initial period where the initial output is consumed) and the investment in capital is always zero. This is ruled out by A.14-A.17 in Yano (1984). Yano (1990) assumes continuous differentiability of the production function (A.1), utility function (A.5), and Inada conditions on the utility function (A.7). There is also an interiority condition ( p.37) that says that all countries (firms) produce a positive output in equilibrium. Our paper does not use these conditions. In fact, in Example 4.2 we show under these conditions it is possible in a competitive equilibrium while there is positive output it is entirely consumed. Yano (1998) also assumes continuous differentiability and Inada conditions for utility (Assumption 1) and production functions (Assumption 2), which are not assumed in our paper.

There are other abstract proofs for existence of a competitive equilibrium in a neoclassical growth model, such as Aliprantis, et al. (1997) which, in principle, could be adapted to show existence in a model with endogenous labor-leisure choice. There are two difficulties in using their approach for the model with endogenous labor-leisure. First, their approach prices the consumption good but it does not directly the equilibrium wage sequence. Second, they assume that the production function is strictly concave and satisfies the Inada condition, $f^{\prime}(0)=\infty$ (p. 670). Their proof relies on showing that free disposal trajectory lies in a compact set (in the appropriate topology) (Lemma 3, p. 672). We do not rely on this argument, thus, we are able to dispense with both of their assumptions. We give examples where there is a competitive equilibrium where the assumptions of this paper are violated (see 4.1-4.3).

The organization of the paper is as follows. Section 2 describes the model. In section 3, we provides the sufficient conditions on the objective function and the constraint functions so that Lagrangean multipliers can be represented by an $\ell_{+}^{1}$ sequence of multipliers in optimal growth model with leisure in the utility function and prove the main result on existence of competitive equilibrium in a model with a representative agent by using these multipliers as sequences of prices and wages. Section 4 gives examples with corner 
solutions to illustrate that a competitive equilibrium will still exist using the main result of the paper. Section 5 concludes.

\section{The model}

We study the optimal growth model with an endogenous labor-leisure choice. Thus, it is an economy where the representative consumer has preferences defined over processes of consumption and leisure described by the utility function

$$
\sum_{t=0}^{\infty} \beta^{t} u\left(c_{t}, l_{t}\right) .
$$

In each period, the consumer faces two resource constraints given by

$$
\begin{aligned}
c_{t}+k_{t+1} & \leq F\left(k_{t}, L_{t}\right)+(1-\delta) k_{t}, \\
l_{t}+L_{t} & =1, \forall t
\end{aligned}
$$

where $F$ is the production function, $\delta \in(0,1)$ is the depreciation rate of capital stock, $l_{t}$ is leisure and $L_{t}$ is labor. These constraints restrict allocations of commodities and time for the leisure.

Formally, the problem of the representative consumer is stated as follows:

$$
\begin{aligned}
& \max \sum_{t=0}^{\infty} \beta^{t} u\left(c_{t}, l_{t}\right) \\
& \text { s.t. } c_{t}+k_{t+1} \leq F\left(k_{t}, 1-l_{t}\right)+(1-\delta) k_{t}, \forall t \geq 0 \\
& c_{t} \geq 0, k_{t} \geq 0, l_{t} \geq 0,1-l_{t} \geq 0, \quad \forall t \geq 0 \\
& k_{0} \geq 0 \text { is given. }
\end{aligned}
$$

We make a set of assumptions on preferences and the production technology. The assumptions on the period utility function $u: \mathbb{R}_{+}^{2} \rightarrow \mathbb{R}$ are:

Assumption U1: $u$ is continuous, concave, increasing on $\mathbb{R}_{+}^{2}$ and strictly increasing on $\mathbb{R}_{++}^{2}$.

It is worth discussing this assumption. In the optimal growth model where there is no labor-leisure choice, the conventional assumption on the one-period utility function is $u$ is strictly increasing in $R_{+}$(Aliprantis, et al (1997), p. 673, and Le Van and Saglam (2004), Assumption 3, p. 400). This does not extend to our model and thus, we assume $u$ to be 
increasing in $R_{+}^{27}$.

Assumption U2: $u(0,0)=0$.

The assumptions on the production function $F: \mathbb{R}_{+}^{2} \rightarrow \mathbb{R}_{+}$are as follows:

Assumption F1: $F$ is continuous, concave, increasing on $\mathbb{R}_{+}^{2}$ and strictly increasing on $\mathbb{R}_{++}^{2}$.

Assumption F2: $F(0,0)=0, \lim _{k \rightarrow 0} F_{k}(k, 1)>\delta, \lim _{k \rightarrow+\infty} F_{k}(k, 1)<\delta$.

Note there is an abuse of notation as by $F_{k}$ we mean the subdifferential of $F$ with respect to $k$. In the rest of the paper, the notation for the partial should be interpreted as the subdifferential as well.

The assumptions U1, U2, F1 are standard. Note we do not assume strict concavity, differentiability or Inada conditions for the utility and production functions. Assumption F2 is a weak assumption to ensure that there is a maximum sustainable capital stock, and thus the sequence of capital is bounded.

We have relaxed some important assumptions in the literature. Bewley (1972) assumes that the production set is a convex cone (Theorem 3). Bewley (1982) assumes the strictly positiveness of derivatives of utility functions on $\mathbb{R}_{+}^{L}$ (strictly monotonicity assumption). In our model, the utility functions may not be differentiable in $\mathbb{R}_{+}^{2}{ }^{8}$ Le Van, et al. (2007) assumed the cross-partial derivative $u_{c l}^{i}$ has constant sign, $u_{c}^{i}(x, x)$ and $u_{l}^{i}(x, x)$ are nonincreasing in $x$, production function $F$ is homogenous of degree $\alpha \leq 1$ and $F_{k L} \geq 0$ (Assumptions U4, F4, U5, F5). We also do not assume Inada conditions (see for example, Goenka, et al. 2012), or $\lim _{\epsilon \rightarrow 0} \frac{u(\epsilon, \epsilon)}{\epsilon} \rightarrow+\infty$ as in Le Van and Vailakis (2004). The assumptions of Aliprantis, et al. (1997) are weakened. In particular, we do not assume that production function is strictly concave and satisfy Inada condition, $f^{\prime}(0)=\infty$ (p. 670 ), and do not assume that $u$ is strictly increasing everywhere. The assumptions of Yano (1984) A.14-A.17 are dropped that require interior consumption, that consumption can be decreased by a certain percentage, uniform bounds on marginal products, and

\footnotetext{
${ }^{7}$ Let $x, y$ be two vectors of $R^{n}$. We write $x \leq y$ if $x_{i} \leq y_{i}$ for all $i$, and $x<y$ if $x_{i} \leq y_{i}$ for all $i$ and $x_{i}<y_{i}$ for at least one $i$.

A function $u(x)$ is said to be increasing if $u(x) \leq u(y)$ for all $x<y$. It is said to be strictly increasing if $u(x)<u(y)$ for all $x<y$.

Consider the standard function of the Cobb-Douglas class: $u(c, l)=\sqrt{c l}$. Let $x(c, l)=(1,0), y(c, l)=$ $(2,0)$. Obviously, $x<y$. However, $u(x)=u(y)=0$. Thus $u(c, l)=\sqrt{c l}$ is increasing on $R_{+}^{2}$ and strictly increasing on $R_{++}^{2}$ but not strictly increasing on $R_{+}^{2}$.

${ }^{8}$ Let $F(k, L)=k^{\alpha} L^{1-\alpha}, \alpha \in(0,1)$. This function is not differentiable even in the extended real numbers at $(0, L)$ or $(k, 0)$ for $L \geq 0, K \geq 0$. The assumptions in Bewley (1982) that $u_{c}>>0, u_{l}>>0$, and $D^{2} u$ is negative definite on $R_{+}^{2}$ are obviously violated.
} 
substitutability on inputs. The assumptions of Yano $(1990,1998)$ on continuous differentiability of utility and production, Inada conditions and interiority of the allocation are dropped. The restrictions on cross-partial derivatives in Aiyagari, et al. (1992), Coleman (1997), Datta, et al. (2002), Greenwood and Huffman (1995) are also not necessary.

We say that a sequence $\left\{c_{t}, k_{t}, l_{t}\right\}_{t=0,1, \ldots, \infty}$ is feasible from $k_{0}$ if it satisfies the constraints

$$
\begin{aligned}
c_{t}+k_{t+1} & \leq F\left(k_{t}, 1-l_{t}\right)+(1-\delta) k_{t}, \quad \forall t \geq 0, \\
c_{t} & \geq 0, k_{t} \geq 0, l_{t} \geq, 1-l_{t} \geq 0, \quad \forall t \geq 0, \\
k_{0} & >0 \text { is given. }
\end{aligned}
$$

It is easy to check that, for any initial condition $k_{0}>0$, a sequence $\mathbf{k}=\left\{k_{t}\right\}_{t=0}^{\infty}$ is feasible iff $0 \leq k_{t+1} \leq F\left(k_{t}, 1\right)+(1-\delta) k_{t}$ for all $t$. The class of feasible capital paths is denoted by $\Pi\left(k_{0}\right)$. A pair of consumption-leisure sequences $\{\mathbf{c}, \mathbf{l}\}=\left\{c_{t}, l_{t}\right\}_{t=0}^{\infty}$ is feasible from $k_{0}>0$ if there exists a sequence $\mathbf{k} \in \Pi\left(k_{0}\right)$ that satisfies $0 \leq c_{t}+k_{t+1} \leq F\left(k_{t}, 1-l_{t}\right)+(1-\delta) k_{t}$ and $0 \leq l_{t} \leq 1$ for all $t$.

Define $f\left(k_{t}, L_{t}\right)=F\left(k_{t}, L_{t}\right)+(1-\delta) k_{t}$. Assumption $\mathbf{F 2}$ implies that

$$
\begin{aligned}
f_{k}(+\infty, 1) & =F_{k}(+\infty, 1)+(1-\delta)<1 \\
f_{k}(0,1) & =F_{k}(0,1)+(1-\delta)>1 .
\end{aligned}
$$

From above, it follows that there exists $\bar{k}>0$ such that: (i) $f(\bar{k}, 1)=\bar{k}$, (ii) $k>\bar{k}$ implies $f(k, 1)<k$, (iii) $k<\bar{k}$ implies $f(k, 1)>k$. Therefore, for any $\mathbf{k} \in \Pi\left(k_{0}\right)$, we have $0 \leq k_{t} \leq \max \left(k_{0}, \bar{k}\right)$. Thus, $\mathbf{k} \in \ell_{+}^{\infty}$ which in turn implies $\mathbf{c} \in \ell_{+}^{\infty}$, if $\{\mathbf{c}, \mathbf{k}\}$ is feasible from $k_{0}$.

\section{Competitive equilibrium}

The definition of a competitive equilibrium is standard.

Definition 1. A competitive equilibrium consists of an allocation $\left\{\mathbf{c}^{*}, \mathbf{l}^{*}, \mathbf{k}^{*}, \mathbf{L}^{*}\right\} \in \ell_{+}^{\infty} \times$ $\ell_{+}^{\infty} \times \ell_{+}^{\infty} \times \ell_{+}^{\infty}$, a price sequence $\mathbf{p}^{*} \in \ell_{+}^{1}$ for the consumption good, a wage sequence $\mathbf{w}^{*} \in \ell_{+}^{1}$ for labor and a price $r>0$ for the initial capital stock $k_{0}$ such that:

i) $\left\{\mathbf{c}^{*}, \mathbf{l}^{*}\right\}$ is a solution to the problem

$$
\begin{array}{ll}
\max & \sum_{t=0}^{\infty} \beta^{t} u\left(c_{t}, l_{t}\right) \\
\text { s.t. } & \mathbf{p}^{*} \mathbf{c} \leq \mathbf{w}^{*} \mathbf{L}+\pi^{*}+r k_{0}
\end{array}
$$


where $\pi^{*}$ is the maximum profit of the firm.

ii) $\left\{\mathbf{k}^{*}, \mathbf{L}^{*}\right\}$ is a solution to the firm's problem

$$
\begin{aligned}
& \pi^{*}=\max \sum_{t=0}^{\infty} p_{t}^{*}\left[f\left(k_{t}, L_{t}\right)-k_{t+1}\right]-\sum_{t=0}^{\infty} w_{t}^{*} L_{t}-r k_{0} \\
\text { s.t. } \quad & 0 \leq k_{t+1} \leq f\left(k_{t}, L_{t}\right), L_{t} \geq 0, \forall t .
\end{aligned}
$$

iii) Markets clear

$$
\begin{aligned}
c_{t}^{*}+k_{t+1}^{*} & =f\left(k_{t}^{*}, L_{t}^{*}\right) \forall t \\
l_{t}^{*}+L_{t}^{*} & =1 \forall t \\
\text { and } k_{0}^{*} & =k_{0}
\end{aligned}
$$

In the following, we show that under maintained assumptions, there exist multipliers of the social planner problem that are summable. We then show that the appropriately chosen multipliers constitute a system of competitive equilibrium prices. The results on existence of a competitive equilibrium in the optimal growth model with inelastic labor supply do not extend immediately to the case of endogenous labor-leisure choice. The difficulty is that the previous results, e.g. Le Van and Vailakis (2004) rely on showing that the allocation is interior as the price of the good is the discounted marginal utility of consumption (See Remark 3 and Theorem 1 in that paper). As we show in the examples in the next section, under our assumptions, a competitive equilibrium can exist even if the capital stock is zero or if the consumption is zero so that the price system in Le Van and Vailakis (2004) is not defined.

We first extend the result of Le Van and Saglam (2004) to the case of endogenous labor to show existence of multipliers. As in that paper and Dechert (1982), we impose conditions on the asymptotic properties on the constraint set that are weaker than Mackey continuity (see conditions $\mathbf{T 1}, \mathbf{T} 2$ and the discussion in Appendix 1).

Proposition 1. If $\mathbf{x}^{*}=\left(\mathbf{c}^{*}, \mathbf{k}^{*}, \mathbf{l}^{*}\right)$ is a solution to the following problem ${ }^{9}$ :

$$
\min -\sum_{t=0}^{\infty} \beta^{t} u\left(c_{t}, l_{t}\right)
$$

\footnotetext{
${ }^{9} \mathrm{~A}$ solution exists following a standard argument which is sketched for completeness. Observe that the feasible set is in a fixed ball of $\ell^{\infty}$ which is weak ${ }^{*}-\left(\ell^{\infty}, \ell^{1}\right)$ compact. We show that the function $\sum_{t=0}^{\infty} \beta^{t} u\left(c_{t}, l_{t}\right)$ is continuous in this topology on the feasible set. Since the weak* topology is metrizable on any ball, we can take a feasible sequence $\left(c_{t}(n), l_{t}(n)\right)_{n}$ converging to some $\left(c_{t}, l_{t}\right)$ in the feasible set. Since any feasible consumptions sequence is uniformly bounded by a number depending only on $k_{0}$, for any $\epsilon>0$ there exists $T_{0}$ such that for any $T \geq T_{0}$, for any $n$, we have

$$
\sum_{t \geq T} \beta^{t} u\left(c_{t}(n), l_{t}(n)\right) \leq \epsilon, \sum_{t \geq T} \beta^{t} u\left(c_{t}, l_{t}\right) \leq \epsilon
$$
}




$$
\begin{aligned}
& \text { s.t. } c_{t}+k_{t+1}-f\left(k_{t}, 1-l_{t}\right) \leq 0 \\
& -c_{t} \leq 0,-k_{t} \leq 0,0 \leq l_{t} \leq 1
\end{aligned}
$$

then there exists $\lambda=\left(\lambda^{1}, \lambda^{2}, \lambda^{3}, \lambda^{4}, \lambda^{5}\right) \in\left(\ell_{+}^{\infty} \times \ell_{+}^{\infty} \times \ell_{+}^{\infty} \times \ell_{+}^{\infty} \times \ell_{+}^{\infty}\right)^{\prime}$, (the dual space of $\left.\ell_{+}^{\infty} \times \ell_{+}^{\infty} \times \ell_{+}^{\infty} \times \ell_{+}^{\infty} \times \ell_{+}^{\infty}\right), \lambda \neq \mathbf{0}$ such that: $\forall \mathbf{x}=(\mathbf{c}, \mathbf{k}, \mathbf{l}) \in \ell_{+}^{\infty} \times \ell_{+}^{\infty} \times \ell_{+}^{\infty}$

$$
\begin{gathered}
\sum_{t=0}^{\infty} \beta^{t} u\left(c_{t}^{*}, l_{t}^{*}\right)-\sum_{t=0}^{\infty} \lambda_{t}^{1}\left(c_{t}^{*}+k_{t+1}^{*}-f\left(k_{t}^{*}, 1-l_{t}^{*}\right)\right) \\
+\sum_{t=0}^{\infty} \lambda_{t}^{2} c_{t}^{*}+\sum_{t=0}^{\infty} \lambda_{t}^{3} k_{t}^{*}+\sum_{t=0}^{\infty} \lambda_{t}^{4} l_{t}^{*}+\sum_{t=0}^{\infty} \lambda_{t}^{5}\left(1-l_{t}^{*}\right) \\
\geq \sum_{t=0}^{\infty} \beta^{t} u\left(c_{t}, l_{t}\right)-\sum_{t=0}^{\infty} \lambda_{t}^{1}\left(c_{t}+k_{t+1}-f\left(k_{t}, 1-l_{t}\right)\right) \\
+\sum_{t=0}^{\infty} \lambda_{t}^{2} c_{t}+\sum_{t=0}^{\infty} \lambda_{t}^{3} k_{t}+\sum_{t=0}^{\infty} \lambda_{t}^{4} l_{t}+\sum_{t=0}^{\infty} \lambda_{t}^{5}\left(1-l_{t}\right) \\
\lambda_{t}^{1}\left(c_{t}^{*}+k_{t+1}^{*}-f\left(k_{t}^{*}, 1-l_{t}^{*}\right)\right)=0, \quad \forall t \geq 0 \\
\lambda_{t}^{2} c_{t}^{*}=0, \quad \forall t \geq 0 \\
\lambda_{t}^{3} k_{t}^{*}=0, \quad \forall t \geq 0 \\
\lambda_{t}^{4} l_{t}^{*}=0, \quad \forall t \geq 0 \\
\lambda_{t}^{5}\left(1-l_{t}^{*}\right)=0, \quad \forall t \geq 0 \\
0 \in \beta^{t} \partial_{2} u\left(c_{t}^{*}, l_{t}^{*}\right)-\lambda_{t}^{1} \partial_{2} f\left(k_{t}^{*}, L_{t}^{*}\right)+\left\{\lambda_{t}^{4}\right\}-\left\{\lambda_{t}^{5}\right\}, \quad \forall t \geq 0 \\
0 \in \lambda_{t}^{1} \partial_{1} f\left(k_{t}^{*}, L_{t}^{*}\right)+\left\{\lambda_{t}^{3}\right\}-\left\{\lambda_{t-1}^{1}\right\}, \quad \forall t \geq 0
\end{gathered}
$$

where $\partial_{i} u\left(c_{t}^{*}, l_{t}^{*}\right), \partial_{i} f\left(k_{t}^{*}, L_{t}^{*}\right)$ respectively denote the projection on the $i^{\text {th }}$ component of the subdifferential of the function $u$ at $\left(c_{t}^{*}, l_{t}^{*}\right)$ and the function $f$ at $\left(k_{t}^{*}, L_{t}^{*}\right)$.

Moreover, $\lambda^{1} \in \ell_{+}^{1} \backslash\{0\}$.

Proof. See Appendix.

Hence,

$$
\left|\sum_{t=0}^{+\infty} \beta^{t}\left[u\left(c_{t}(n), l_{t}(n)\right)-u\left(c_{t}, l_{t}\right)\right]\right| \leq \sum_{t=0}^{T-1} \beta^{t}\left|u\left(c_{t}(n), l_{t}(n)\right)-u\left(c_{t}, l_{t}\right)\right|+2 \epsilon .
$$

Since weak* ${ }^{*}$ convergence implies pointwise convergence, the result is established. 
This result, thus, also shows whether an optimal path is interior or not is not important for the existence of an optimal path (See Kamihigashi (2015)). Once we have the existence of non-zero multipliers, we give the main result where we define the price system and show that they are indeed competitive prices.

Theorem 1. Let $\left\{\mathbf{c}^{*}, \mathbf{k}^{*}, \mathbf{l}^{*}\right\}$ solve Problem $(Q)$. Take

$$
p_{t}^{*}=\lambda_{t}^{1} \text { for any } t \text { and } r>0 \text {. }
$$

There exists $f_{L}\left(k_{t}^{*}, L_{t}^{*}\right) \in \partial_{2} f\left(k_{t}^{*}, L_{t}^{*}\right)$ such that $\left\{\mathbf{c}^{*}, \mathbf{k}^{*}, \mathbf{L}^{*}, \mathbf{p}^{*}, \mathbf{w}^{*}, r\right\}$ is a competitive equilibrium with $w_{t}^{*}=\lambda_{t}^{1} f_{L}\left(k_{t}^{*}, L_{t}^{*}\right)$.

Proof. Consider $\lambda=\left\{\lambda^{\mathbf{1}}, \lambda^{\mathbf{2}}, \lambda^{\mathbf{3}}, \lambda^{\mathbf{4}}, \lambda^{\mathbf{5}}\right\}$ of Proposition 1. Conditions (7), (8), (9) in Proposition 1 show that $\partial u\left(c_{t}^{*}, l_{t}^{*}\right)$ and $\partial f\left(k_{t}^{*}, L_{t}^{*}\right)$ are nonempty and there exist $u_{c}\left(c_{t}^{*}, l_{t}^{*}\right) \in$ $\partial_{1} u\left(c_{t}^{*}, l_{t}^{*}\right), u_{l}\left(c_{t}^{*}, l_{t}^{*}\right) \in \partial_{2} u\left(c_{t}^{*}, l_{t}^{*}\right), f_{k}\left(k_{t}^{*}, L_{t}^{*}\right) \in \partial_{1} f\left(k_{t}^{*}, L_{t}^{*}\right)$ and $f_{L}\left(k_{t}^{*}, L_{t}^{*}\right) \in \partial_{2} f\left(k_{t}^{*}, L_{t}^{*}\right)$ such that $\forall t$

$$
\begin{gathered}
\beta^{t} u_{c}\left(c_{t}^{*}, l_{t}^{*}\right)-\lambda_{t}^{1}+\lambda_{t}^{2}=0 \\
\beta^{t} u_{l}\left(c_{t}^{*}, l_{t}^{*}\right)-\lambda_{t}^{1} f_{L}\left(k_{t}^{*}, L_{t}^{*}\right)+\lambda_{t}^{4}-\lambda_{t}^{5}=0 \\
\lambda_{t}^{1} f_{k}\left(k_{t}^{*}, L_{t}^{*}\right)+\lambda_{t}^{3}-\lambda_{t-1}^{1}=0
\end{gathered}
$$

Define $w_{t}^{*}=\lambda_{t}^{1} f_{L}\left(k_{t}^{*}, L_{t}^{*}\right)<+\infty$.

First, we claim that $\mathbf{w}^{*} \in \ell_{+}^{1}$.

We have

$$
+\infty>\sum_{t=0}^{\infty} \beta^{t} u\left(c_{t}^{*}, l_{t}^{*}\right)-\sum_{t=0}^{\infty} \beta^{t} u(0,0) \geq \sum_{t=0}^{\infty} \beta^{t} u_{c}\left(c_{t}^{*}, l_{t}^{*}\right) c_{t}^{*}+\sum_{t=0}^{\infty} \beta^{t} u_{l}\left(c_{t}^{*}, l_{t}^{*}\right) l_{t}^{*},
$$

which implies

$$
\sum_{t=0}^{\infty} \beta^{t} u_{l}\left(c_{t}^{*}, l_{t}^{*}\right) l_{t}^{*}<+\infty
$$

and

$$
+\infty>\sum_{t=0}^{\infty} \lambda_{t}^{1} f\left(k_{t}^{*}, L_{t}^{*}\right)-\sum_{t=0}^{\infty} \lambda_{t}^{1} f(0,0) \geq \sum_{t=0}^{\infty} \lambda_{t}^{1} f_{k}\left(k_{t}^{*}, L_{t}^{*}\right) k_{t}^{*}+\sum_{t=0}^{\infty} \lambda_{t}^{1} f_{L}\left(k_{t}^{*}, L_{t}^{*}\right) L_{t}^{*}
$$

which implies

$$
\sum_{t=0}^{\infty} \lambda_{t}^{1} f_{L}\left(k_{t}^{*}, L_{t}^{*}\right) L_{t}^{*}<+\infty
$$


Given $T$, we multiply (11) by $L_{t}^{*}$ and sum up from 0 to $T$. Observe that

$$
\begin{aligned}
\forall T, \sum_{t=0}^{T} \beta^{t} u_{l}\left(c_{t}^{*}, l_{t}^{*}\right) L_{t}^{*} & =\sum_{t=0}^{T} \lambda_{t}^{1} f_{L}\left(k_{t}^{*}, L_{t}^{*}\right) L_{t}^{*}+\sum_{t=0}^{T} \lambda_{t}^{5} L_{t}^{*}-\sum_{t=0}^{T} \lambda_{t}^{4} L_{t}^{*} . \\
0 & \leq \sum_{t=0}^{\infty} \lambda_{t}^{5} L_{t}^{*} \leq \sum_{t=0}^{\infty} \lambda_{t}^{5}<+\infty . \\
0 & \leq \sum_{t=0}^{\infty} \lambda_{t}^{4} L_{t}^{*} \leq \sum_{t=0}^{\infty} \lambda_{t}^{4}<+\infty .
\end{aligned}
$$

Thus, since $L_{t}^{*}=1-l_{t}^{*}$, from $(15)$, we get

$$
\begin{aligned}
\sum_{t=0}^{T} \beta^{t} u_{l}\left(c_{t}^{*}, l_{t}^{*}\right)= & \sum_{t=0}^{T} \beta^{t} u_{l}\left(c_{t}^{*}, l_{t}^{*}\right) l_{t}^{*}+\sum_{t=0}^{T} \lambda_{t}^{1} f_{L}\left(k_{t}^{*}, L_{t}^{*}\right) L_{t}^{*} \\
& +\sum_{t=0}^{T} \lambda_{t}^{5} L_{t}^{*}-\sum_{t=0}^{T} \lambda_{t}^{4} L_{t}^{*} .
\end{aligned}
$$

Using (13), (14), (16), (17) and letting $T \rightarrow \infty$, we obtain

$$
\begin{aligned}
0 \leq & \sum_{t=0}^{\infty} \beta^{t} u_{l}\left(c_{t}^{*}, l_{t}^{*}\right)=\sum_{t=0}^{\infty} \beta^{t} u_{l}\left(c_{t}^{*}, l_{t}^{*}\right) l_{t}^{*}+\sum_{t=0}^{\infty} \lambda_{t}^{1} f_{L}\left(k_{t}^{*}, L_{t}^{*}\right) L_{t}^{*} \\
& +\sum_{t=0}^{\infty} \lambda_{t}^{5} L_{t}^{*}-\sum_{t=0}^{\infty} \lambda_{t}^{4} L_{t}^{*}<+\infty .
\end{aligned}
$$

Consequently, from (11), $\sum_{t=0}^{\infty} \lambda_{t}^{1} f_{L}\left(k_{t}^{*}, L_{t}^{*}\right)<+\infty$ i.e. $\mathbf{w}^{*} \in \ell_{+}^{1}$. So, we have $\left\{\mathbf{c}^{*}, \mathbf{l}^{*}, \mathbf{k}^{*}, \mathbf{L}^{*}\right\} \in$ $\ell_{+}^{\infty} \times \ell_{+}^{\infty} \times \ell_{+}^{\infty} \times \ell_{+}^{\infty}$, with $\mathbf{p}^{*} \in \ell_{+}^{1}$ and $\mathbf{w}^{*} \in \ell_{+}^{1}$.

We now show that $\left(\mathbf{k}^{*}, \mathbf{L}^{*}\right)$ is solution to the firm's problem.

Since $p_{t}^{*}=\lambda_{t}^{1}, w_{t}^{*}=\lambda_{t}^{1} f_{L}\left(k_{t}^{*}, L_{t}^{*}\right)$, we have

$$
\pi^{*}=\sum_{t=0}^{\infty} \lambda_{t}^{1}\left[f\left(k_{t}^{*}, L_{t}^{*}\right)-k_{t+1}^{*}\right]-\sum_{t=0}^{\infty} \lambda_{t}^{1} f_{L}\left(k_{t}^{*}, L_{t}^{*}\right) L_{t}^{*}-r k_{0}
$$

Let :

$$
\begin{aligned}
\Delta_{T}= & \sum_{t=0}^{T} \lambda_{t}^{1}\left[f\left(k_{t}^{*}, L_{t}^{*}\right)-k_{t+1}^{*}\right]-\sum_{t=0}^{T} \lambda_{t}^{1} f_{L}\left(k_{t}^{*}, L_{t}^{*}\right) L_{t}^{*}-r k_{0} \\
& -\left(\sum_{t=0}^{T} \lambda_{t}^{1}\left[f\left(k_{t}, L_{t}\right)-k_{t+1}\right]-\sum_{t=0}^{T} \lambda_{t}^{1} f_{L}\left(k_{t}, L_{t}\right) L_{t}-r k_{0}\right) .
\end{aligned}
$$


From the concavity of $f$, we get

$$
\begin{aligned}
\Delta_{T} \geq & \sum_{t=1}^{T} \lambda_{t}^{1} f_{k}\left(k_{t}^{*}, L_{t}^{*}\right)\left(k_{t}^{*}-k_{t}\right)-\sum_{t=0}^{T} \lambda_{t}^{1}\left(k_{t+1}^{*}-k_{t+1}\right) \\
= & {\left[\lambda_{1}^{1} f_{k}\left(k_{1}^{*}, L_{1}^{*}\right)-\lambda_{0}^{1}\right]\left(k_{1}^{*}-k_{1}\right)+\ldots } \\
& +\left[\lambda_{T}^{1} f_{k}\left(k_{T}^{*}, L_{T}^{*}\right)-\lambda_{T-1}^{1}\right]\left(k_{T}^{*}-k_{T}\right)-\lambda_{T}^{1}\left(k_{T+1}^{*}-k_{T+1}\right) .
\end{aligned}
$$

By (4) and (12), we have: $\forall t=1,2, \ldots, T$

$$
\left[\lambda_{t}^{1} f_{k}\left(k_{t}^{*}, L_{t}^{*}\right)-\lambda_{t-1}^{1}\right]\left(k_{t}^{*}-k_{t}\right)=-\lambda_{t}^{3}\left(k_{t}^{*}-k_{t}\right)=\lambda_{t}^{3} k_{t} \geq 0 .
$$

Thus,

$$
\Delta_{T} \geq-\lambda_{T}^{1}\left(k_{T+1}^{*}-k_{T+1}\right)=-\lambda_{T}^{1} k_{T+1}^{*}+\lambda_{T}^{1} k_{T+1} \geq-\lambda_{T}^{1} k_{T+1}^{*} .
$$

Since $\lambda^{1} \in \ell_{+}^{1}, \sup _{T} k_{T+1}^{*}<+\infty$, we have

$$
\lim _{T \rightarrow+\infty} \Delta_{T} \geq \lim _{T \rightarrow+\infty}-\lambda_{T}^{1} k_{T+1}^{*}=0
$$

We have proved that the sequences $\left(\mathbf{k}^{*}, \mathbf{L}^{*}\right)$ maximize the profit of the firm. We now show that $\mathbf{c}^{*}$ solves the consumer's problem.

$$
\text { Let }\{\mathbf{c}, \mathbf{L}\} \text { satisfy } \sum_{t=0}^{\infty} \lambda_{t}^{1} c_{t} \leq \sum_{t=0}^{\infty} w_{t}^{*} L_{t}+\pi^{*}+r k_{0}
$$

By the concavity of $u$, we have:

$$
\begin{gathered}
\Delta=\sum_{t=0}^{\infty} \beta^{t} u\left(c_{t}^{*}, l_{t}^{*}\right)-\sum_{t=0}^{\infty} \beta^{t} u\left(c_{t}, l_{t}\right) \\
\geq \sum_{t=0}^{\infty} \beta^{t} u_{c}\left(c_{t}^{*}, l_{t}^{*}\right)\left(c_{t}^{*}-c_{t}\right)+\sum_{t=0}^{\infty} \beta^{t} u_{l}\left(c_{t}^{*}, l_{t}^{*}\right)\left(l_{t}^{*}-l_{t}\right) .
\end{gathered}
$$

Combining (3), (6), (10), (11) yields

$$
\begin{gathered}
\Delta \geq \sum_{t=0}^{\infty}\left(\lambda_{t}^{1}-\lambda_{t}^{2}\right)\left(c_{t}^{*}-c_{t}\right)+\sum_{t=0}^{\infty}\left(\lambda_{t}^{1} f_{L}\left(k_{t}^{*}, 1-l_{t}^{*}\right)+\lambda_{t}^{5}-\lambda_{t}^{4}\right)\left(l_{t}^{*}-l_{t}\right) \\
=\sum_{t=0}^{\infty} \lambda_{t}^{1}\left(c_{t}^{*}-c_{t}\right)+\sum_{t=0}^{\infty} \lambda_{t}^{2} c_{t}-\sum_{t=0}^{\infty} \lambda_{t}^{2} c_{t}^{*}+\sum_{t=0}^{\infty}\left(w_{t}^{*}+\lambda_{t}^{5}\right)\left(l_{t}^{*}-l_{t}\right) \\
-\sum_{t=0}^{\infty} \lambda_{t}^{4} l_{t}^{*}+\sum_{t=0}^{\infty} \lambda_{t}^{4} l_{t}
\end{gathered}
$$




$$
\begin{aligned}
\geq & \sum_{t=0}^{\infty} \lambda_{t}^{1}\left(c_{t}^{*}-c_{t}\right)+\sum_{t=0}^{\infty}\left(w_{t}^{*}+\lambda_{t}^{5}\right)\left(l_{t}^{*}-l_{t}\right)= \\
& \sum_{t=0}^{\infty} \lambda_{t}^{1}\left(c_{t}^{*}-c_{t}\right)+\sum_{t=0}^{\infty} w_{t}^{*}\left(l_{t}^{*}-l_{t}\right)+\sum_{t=0}^{\infty} \lambda_{t}^{5}\left(1-l_{t}\right) \\
\geq & \sum_{t=0}^{\infty} \lambda_{t}^{1}\left(c_{t}^{*}-c_{t}\right)+\sum_{t=0}^{\infty} w_{t}^{*}\left(L_{t}-L_{t}^{*}\right) .
\end{aligned}
$$

Since

$$
\pi^{*}=\sum_{t=0}^{\infty} \lambda_{t}^{1} c_{t}^{*}-\sum_{t=0}^{\infty} w_{t}^{*} L_{t}^{*}-r k_{0}
$$

it follows from (18) that

$$
\begin{aligned}
\Delta & \geq \sum_{t=0}^{\infty} p_{t}^{*} c_{t}^{*}-\sum_{t=0}^{\infty} w_{t}^{*} L_{t}^{*}-r k_{0}-\left(\sum_{t=0}^{\infty} p_{t}^{*} c_{t}-\sum_{t=0}^{\infty} w_{t}^{*} L_{t}-r k_{0}\right) \\
& \geq \pi^{*}-\pi^{*}=0
\end{aligned}
$$

Consequently, $\Delta \geq 0$ that means $c^{*}$ solves the consumer's problem.

Finally, the market clears at every period, since $\forall t, c_{t}^{*}+k_{t+1}^{*}=f\left(k_{t}^{*}, L_{t}^{*}\right)$ and $1-l_{t}^{*}=$ $L_{t}^{*}$.

\section{Examples}

We give three parametric example illustrating generality of our result. In these examples there are corner solutions that the literature makes assumptions to rule out. In each of the examples the competitive equilibrium is calculated. They illustrate that the interiority of an allocation is not necessary for existence of a competitive equilibrium.

In the first example, there is a competitive equilibrium with zero labor supply, the good being produced through capital alone. As a consumer may choose to enjoy all available time as leisure, imposing an Inada condition on productivity of labor is not well justified. As we show in this case we still have existence of competitive equilibria.

In the second example, we show that a competitive equilibrium will exist even if $k_{t}=$ $0, \forall t \geq 1$. Thus, showing that the capital stock is positive is not necessary for existence. In this example, the good is produced through labor alone, and consumption of both the good and leisure is positive.

The third example, shows that it can be the case that $k_{t}=c_{t}=L_{t}=0, \forall t \geq 1$, that is the

consumer just consumes leisure. In this case, the price system as in Le Van and Saglam 
(2004) and Le Van and Vailakis (2004) is not defined but we can still show existence of a competitive equilibrium. The conditions of Aliprantis, et al. (1997) also do not apply.

\subsection{Example 1: Competitive equilibrium with $L_{t}^{*}=0, l_{t}^{*}=1$}

Consider an economy with a good that can either be consumed or invested as capital, one firm and one consumer. The consumer has preferences defined over processes of consumption and leisure described by the utility function

$$
\sum_{t=0}^{\infty} \beta^{t} u\left(c_{t}, l_{t}\right)=\sum_{t=0}^{\infty} \beta^{t}\left(c_{t}+m l_{t}\right)
$$

The firm produces capital good by using capital $k_{t}$ and labor $L_{t}=1-l_{t}$. The production function $f\left(k_{t}, L_{t}\right)=\left(k_{t}^{\alpha}+L_{t}\right)^{1 / \theta}, 0<\alpha<\theta, 0<\beta<1<\theta, f$ is concave and increasing. Assume that $m=\frac{1}{\theta}\left(\frac{\beta \alpha}{\theta}\right)^{\frac{\alpha(1-\theta)}{\theta-\alpha}}$. As we see below, we need impose a condition on $k_{0}$ which is $k_{0}^{\alpha}+1-(\theta m)^{\frac{\theta}{1-\theta}} \in[0,1]^{10}$ to guarantee existence of solutions.

The planning problem is

$$
\begin{aligned}
& \max \sum_{t=0}^{\infty} \beta^{t}\left(c_{t}+m l_{t}\right) \\
\text { s.t. } c_{t}+k_{t+1} \leq & \left(k_{t}^{\alpha}+L_{t}\right)^{1 / \theta}, \forall t \geq 0 \\
L_{t}+l_{t}= & 1, \forall t \geq 0 \\
c_{t} \geq & 0, k_{t} \geq 0, l_{t} \geq 0,1-l_{t} \geq 0, \forall t \geq 0 \\
k_{0} \geq & 0 \text { is given. }
\end{aligned}
$$

Inada conditions are not satisfied for both the utility and production functions. ${ }^{11}$ The utility function is also not strictly concave. We will check both necessary and sufficient conditions.

\section{Necessary condition:}

\footnotetext{
${ }^{10}$ Let us check, for example, $\alpha=1, \beta=\frac{1}{2}, \theta=2$ then $m=2$ and $k_{0}^{\alpha}+1-(\theta m)^{\frac{\theta}{1-\theta}}=k_{0}+\frac{3}{4} \in[0,1]$ for any $0 \leq k_{0} \leq \frac{1}{4}$.

${ }^{11}$ Linearity of the utility function in consumption is not important for the two examples. This, however, simplifies the calculations.
} 
Let $\lambda_{t}=\left(\lambda_{t}^{i}\right)_{i=1}^{5}, \lambda_{t} \neq 0$ denote the Lagrange multipliers. The Lagrangean is

$$
\begin{aligned}
\mathcal{H}= & \sum_{t=0}^{\infty} \beta^{t} u\left(c_{t}, l_{t}\right)-\sum_{t=0}^{\infty} \lambda_{t}^{1}\left(c_{t}+k_{t+1}-f\left(k_{t}, 1-l_{t}\right)\right) \\
& +\sum_{t=0}^{\infty} \lambda_{t}^{2} c_{t}+\sum_{t=0}^{\infty} \lambda_{t}^{3} k_{t}+\sum_{t=0}^{\infty} \lambda_{t}^{4} l_{t}+\sum_{t=0}^{\infty} \lambda_{t}^{5}\left(1-l_{t}\right)
\end{aligned}
$$

It follows from Kuhn-Tucker necessary conditions that, $\forall t \geq 0$

$$
\begin{aligned}
0 & =\beta^{t}-\lambda_{t}^{1}+\lambda_{t}^{2} \\
0 & =\beta^{t} m-\frac{1}{\theta} \lambda_{t}^{1}\left(k_{t}^{\alpha}+1-l_{t}\right)^{\frac{1-\theta}{\theta}}+\lambda_{t}^{4}-\lambda_{t}^{5} \\
0 & =\frac{\alpha}{\theta} \lambda_{t+1}^{1} k_{t+1}^{\alpha-1}\left(k_{t+1}^{\alpha}+1-l_{t+1}\right)^{\frac{1-\theta}{\theta}}+\lambda_{t+1}^{3}-\lambda_{t}^{1} \\
0 & =\lambda_{t}^{1}\left(c_{t}+k_{t+1}-\left(k_{t}^{\alpha}+L_{t}\right)^{1 / \theta}\right) \\
\lambda_{t}^{2} c_{t} & =0, \lambda_{t}^{3} k_{t}=0, \lambda_{t}^{4} l_{t}=0, \lambda_{t}^{5}\left(1-l_{t}\right)=0 .
\end{aligned}
$$

It is easy to check that, the above system of equation has a solution :

$$
\begin{aligned}
\lambda_{t}^{* 1} & =\beta^{t}, \lambda_{t}^{* 2}=\lambda_{t}^{* 3}=\lambda_{t}^{* 4}=\lambda_{t}^{* 5}=0, \\
k_{0}^{*} & =k_{0}, k_{t}^{*}=\left(\frac{\beta \alpha}{\theta}\right)^{\frac{\theta}{\theta-\alpha}}:=k_{s} \in(0,1) \forall t \geq 1, \\
c_{t}^{*} & =\left(k_{s}\right)^{\alpha / \theta}-k_{s}>0, \\
l_{t}^{*} & =1, \\
L_{t}^{*} & =0 .
\end{aligned}
$$

At $t=0$, we have

$$
\begin{aligned}
\lambda_{0}^{* 1} & =1, \lambda_{0}^{* 2}=\lambda_{0}^{* 3}=\lambda_{0}^{* 4}=\lambda_{0}^{* 5}=0, \\
k_{0}^{*} & =k_{0} \\
l_{0}^{*} & =k_{0}^{\alpha}+1-(\theta m)^{\frac{\theta}{1-\theta}} \in[0,1] \\
L_{0}^{*} & =1-l_{0}^{*}, \\
c_{0}^{*} & =\left(k_{0}^{\alpha}+L_{0}^{*}\right)^{1 / \theta}-k_{s}>0,
\end{aligned}
$$

\section{Sufficient condition:}

The Euler equations (i.e, equations (19) and (20 )) imply

$$
\frac{\partial f\left(k_{t+1}^{*}, L_{t+1}^{*}\right)}{\partial k_{t}}=\frac{1}{\beta}, \frac{\partial f\left(k_{t+1}^{*}, L_{t+1}^{*}\right)}{\partial L_{t}}=m .
$$


Because $f\left(k_{t}, L_{t}\right)$ is concave, we have

$$
\begin{aligned}
f\left(k_{t}^{*}, L_{t}^{*}\right)-f\left(k_{t}, L_{t}\right) & \geq \frac{\partial f\left(k_{t+1}^{*}, L_{t+1}^{*}\right)}{\partial k_{t}}\left(k_{t}^{*}-k_{t}\right)+\frac{\partial f\left(k_{t+1}^{*}, L_{t+1}^{*}\right)}{\partial L_{t}}\left(L_{t}^{*}-L_{t}\right) \\
& =\frac{1}{\beta}\left(k_{t}^{*}-k_{t}\right)+m\left(L_{t}^{*}-L_{t}\right)=\frac{1}{\beta}\left(k_{t}^{*}-k_{t}\right)-m\left(1-l_{t}\right) .
\end{aligned}
$$

Let us consider

$$
\begin{aligned}
\Delta_{T} & =\sum_{t=0}^{T} \beta^{t}\left(c_{t}^{*}+m l_{t}^{*}\right)-\sum_{t=0}^{T} \beta^{t}\left(c_{t}+m l_{t}\right) \\
& =\sum_{t=0}^{T} \beta^{t}\left[\left(c_{t}^{*}-c_{t}\right)+m\left(1-l_{t}\right)\right] \\
& \geq \sum_{t=0}^{T} \beta^{t}\left[f\left(k_{t}^{*}, L_{t}^{*}\right)-f\left(k_{t}, L_{t}\right)-\left(k_{t+1}^{*}-k_{t+1}\right)+m\left(1-l_{t}\right)\right] \\
& \geq \sum_{t=0}^{T} \beta^{t}\left[\frac{\partial f\left(k_{t+1}^{*}, L_{t+1}^{*}\right)}{\partial k_{t}}\left(k_{t}^{*}-k_{t}\right)+\frac{\partial f\left(k_{t+1}^{*}, L_{t+1}^{*}\right)}{\partial L_{t}}\left(L_{t}^{*}-L_{t}\right)-\left(k_{t+1}^{*}-k_{t+1}\right)+m\left(1-l_{t}\right)\right] \\
& =\sum_{t=0}^{T} \beta^{t}\left[\frac{1}{\beta}\left(k_{t}^{*}-k_{t}\right)-\left(k_{t+1}^{*}-k_{t+1}\right)\right] \\
& =\frac{1}{\beta}\left(k_{0}^{*}-k_{0}\right)+\sum_{t=1}^{T} \beta^{t-1}\left(k_{t}^{*}-k_{t}\right)-\sum_{t=0}^{T} \beta^{t}\left[k_{t+1}^{*}-k_{t+1}\right] \\
& =-\beta^{T}\left(k_{T+1}^{*}-k_{T+1}\right) \geq-\beta^{T} k_{T+1}^{*}=-k_{s} \beta^{T} .
\end{aligned}
$$

Therefore $\lim _{T \rightarrow \infty} \Delta_{T} \geq 0$ and

$$
\sum_{t=0}^{\infty} \beta^{t}\left(c_{t}^{*}+m l_{t}^{*}\right) \geq \sum_{t=0}^{\infty} \beta^{t}\left(c_{t}+m l_{t}\right) .
$$

As we show in section 3, if we define the sequence price $p_{t}^{*}=\lambda_{t}^{* 1}=\beta^{t}$ for the consumption good and $w_{t}^{*} \in \lambda_{t}^{* 1} \partial_{2} f\left(k_{t}^{*}, L_{t}^{*}\right)=\lambda_{t}^{* 1} f_{L}\left(k_{s}, 0\right)=\beta^{t} \frac{1}{\theta} k_{s}^{\frac{\alpha(1-\theta)}{\theta}}$ then $p_{t}^{*} \in \ell_{+}^{1}, w_{t}^{*} \in \ell_{+}^{1}$ and $\left\{\mathbf{c}^{*}, \mathbf{k}^{*}, \mathbf{L}^{*}, \mathbf{p}^{*}, \mathbf{w}^{*}, r\right\}$ is a competitive equilibrium.

\subsection{Example 2: Competitive equilibrium with $k_{t}^{*}=0, t \geq 1$}

Now consider the production function $f\left(k_{t}, L_{t}\right)=\left(k_{t}+L_{t}^{\alpha}\right)^{1 / \theta}$ where $0<\alpha<\theta, 0<\beta<$ $1<\theta$ and the utility function

$$
u\left(c_{t}, l_{t}\right)=c_{t}+\frac{1}{\theta}\left(\frac{\beta}{\theta}\right)^{\frac{\alpha-\theta}{\alpha(\theta-1)}} l_{t}
$$


We obtain the Kuhn-Tucker conditions, $\forall t \geq 0$

$$
\begin{aligned}
0 & =\beta^{t}-\lambda_{t}^{1}+\lambda_{t}^{2} \\
0 & =\frac{1}{\theta}\left(\frac{\beta}{\theta}\right)^{\frac{\alpha-\theta}{\alpha(\theta-1)}} \beta^{t}-\frac{\alpha}{\theta} \lambda_{t}^{1} L_{t}^{\alpha-1}\left(k_{t}+L_{t}^{\alpha}\right)^{\frac{1-\theta}{\theta}}+\lambda_{t}^{4}-\lambda_{t}^{5} \\
0 & =\frac{1}{\theta} \lambda_{t+1}^{1}\left(k_{t+1}+L_{t+1}^{\alpha}\right)^{\frac{1-\theta}{\theta}}+\lambda_{t+1}^{3}-\lambda_{t}^{1} \\
0 & =\lambda_{t}^{1}\left(c_{t}+k_{t+1}-\left(k_{t}+L_{t}^{\alpha}\right)^{1 / \theta}\right) \\
\lambda_{t}^{2} c_{t} & =0, \lambda_{t}^{3} k_{t}=0, \lambda_{t}^{4} l_{t}=0, \lambda_{t}^{5}\left(1-l_{t}\right)=0 .
\end{aligned}
$$

The system of equation has solution $\forall t \geq 1$,

$$
\begin{aligned}
\lambda_{t}^{* 1} & =\beta^{t}, \lambda_{t}^{* 2}=\lambda_{t}^{* 3}=\lambda_{t}^{* 4}=\lambda_{t}^{* 5}=0 \\
k_{t}^{*} & =0 \\
L_{t}^{*} & =\left(\frac{\beta}{\theta}\right)^{\frac{\theta}{\alpha(\theta-1)}}:=L_{s} \in(0,1) \\
c_{t}^{*} & =\left(L_{s}\right)^{\alpha / \theta}>0 \\
l_{t}^{*} & =1-L_{s} .
\end{aligned}
$$

At $t=0$, solutions are determined by

$$
\begin{aligned}
\lambda_{0}^{* 1} & =1, \lambda_{0}^{* 2}=\lambda_{0}^{* 3}=\lambda_{0}^{* 4}=\lambda_{0}^{* 5}=0 \\
k_{0}^{*} & =k_{0}, c_{0}^{*}=\left(k_{0}+L_{0}^{* \alpha}\right)^{1 / \theta}, \\
l_{0}^{*} & =1-L_{0}^{*},
\end{aligned}
$$

where $L_{0}$ is determined by $L_{0}^{* \alpha-1}\left(k_{0}+L_{0}^{* \alpha}\right)^{\frac{1-\theta}{\theta}}=\frac{1}{\alpha}\left(\frac{\beta}{\theta}\right)^{\frac{\alpha-\theta}{\alpha(\theta-1)}} \cdot{ }^{12}$

The argument for showing the sufficient condition is similar to Example 1.

\subsection{Example 3: Competitive equilibrium with $L_{t}^{*}=c_{t}^{*}=k_{t}^{*}=$ $0, l_{t}^{*}=1, t \geq 1$}

In this example, we relax assumption $\mathbf{F} 2$ and allow $\lim _{k \rightarrow \infty} F_{k}(k, 1)>1$. Therefore, the capital can be unbounded and hence, the free disposal feasible set as in Aliprantis, et al. (1997) is not compact. We show that the Lagrange multipliers form an equilibrium sequence of price and wages. Because our theoretical results have not addressed this case in a general setting, we will prove directly that the sequence is an equilibrium in this

\footnotetext{
${ }^{12}$ We can choose appropriate parameters so that this equation has a solution. For example, let $\alpha=$ $1, \beta=\frac{1}{2}, \theta=2$ then the equation has a solution $L_{0}^{*}=\frac{1}{16}-k_{0} \in[0,1]$ for any $k_{0} \in\left[0, \frac{1}{16}\right]$.
} 
simplified example. It highlights the methods of using Lagrange multipliers in this paper can be employed for an extension of literature can potentially be in more general settings than Aliprantis, et al. (1997).

Consider an economy with a good that can either be consumed or invested as capital, one firm and one consumer. The consumer has preferences defined over processes of consumption and leisure described by the utility function

$$
\sum_{t=0}^{\infty} \beta^{t} u\left(c_{t}, l_{t}\right)=\sum_{t=0}^{\infty} \beta^{t}\left(c_{t}+m l_{t}\right),
$$

The firm produces capital good by using capital $k_{t}$ and labor $L_{t}=1-l_{t}$. The production function $f\left(k_{t}, L_{t}\right)=\frac{1}{\beta} k_{t}+m L_{t}, 0<\beta<1,0<m$. The planning problem is

$$
\begin{aligned}
& \max \sum_{t=0}^{\infty} \beta^{t}\left(c_{t}+m l_{t}\right) \\
\text { s.t. } c_{t}+k_{t+1} \leq & \frac{1}{\beta} k_{t}+m L_{t}, \forall t \geq 0 \\
L_{t}+l_{t} & =1, \forall t \geq 0 \\
c_{t} & \geq 0, k_{t} \geq 0, l_{t} \geq 0,1-l_{t} \geq 0, \forall t \geq 0 \\
k_{0} \geq 0 \text { is given. } &
\end{aligned}
$$

The utility and production functions are both linear and the Inada conditions do not hold. Let $\lambda_{t}=\left(\lambda_{t}^{i}\right)_{i=1}^{5}, \lambda_{t} \neq 0$ denote the Lagrange multipliers. The Lagrangean is

$$
\begin{aligned}
\mathcal{H}= & \sum_{t=0}^{\infty} \beta^{t}\left(c_{t}+m l_{t}\right)-\sum_{t=0}^{\infty} \lambda_{t}^{1}\left[c_{t}+k_{t+1}-\frac{1}{\beta} k_{t}-m\left(1-l_{t}\right)\right] \\
& +\sum_{t=0}^{\infty} \lambda_{t}^{2} c_{t}+\sum_{t=0}^{\infty} \lambda_{t}^{3} k_{t}+\sum_{t=0}^{\infty} \lambda_{t}^{4} l_{t}+\sum_{t=0}^{\infty} \lambda_{t}^{5}\left(1-l_{t}\right) .
\end{aligned}
$$

It follows from Kuhn-Tucker necessary conditions that, $\forall t \geq 0$

$$
\begin{aligned}
0 & =\beta^{t}-\lambda_{t}^{1}+\lambda_{t}^{2} \\
0 & =\beta^{t} m-\lambda_{t}^{1} m+\lambda_{t}^{4}-\lambda_{t}^{5} \\
0 & =\frac{1}{\beta} \lambda_{t+1}^{1}-\lambda_{t}^{1}+\lambda_{t+1}^{3} \\
0 & =\lambda_{t}^{1}\left[c_{t}+k_{t+1}-\frac{1}{\beta} k_{t}-m\left(1-l_{t}\right)\right] \\
\lambda_{t}^{2} c_{t} & =0, \lambda_{t}^{3} k_{t}=0, \lambda_{t}^{4} l_{t}=0, \lambda_{t}^{5}\left(1-l_{t}\right)=0 .
\end{aligned}
$$


It is easy to check that, the above system of equation has a solution ${ }^{13}$

$$
\begin{aligned}
\lambda_{t}^{* 1} & =\beta^{t}, \forall t \geq 0 \\
\lambda_{t}^{* 2} & =\lambda_{t}^{* 3}=\lambda_{t}^{* 4}=\lambda_{t}^{* 5}=0, \forall t \geq 0 \\
k_{0}^{*} & =k_{0}, c_{0}^{*}=\frac{k_{0}}{\beta} \\
c_{t}^{*} & =0, \forall t \geq 1 \\
k_{t}^{*} & =0, \forall t \geq 1 \\
L_{t}^{*} & =0, \forall t \geq 0 \\
l_{t}^{*} & =1, \forall t \geq 0 .
\end{aligned}
$$

Let us define the sequence price $p_{t}^{*}=\lambda_{t}^{* 1}=\beta^{t}$ for the consumption good and wage $w_{t}^{*}=m \beta^{t}$. We will show that $\left\{\mathbf{c}^{*}, \mathbf{k}^{*}, \mathbf{L}^{*}, \mathbf{p}^{*}, \mathbf{w}^{*}\right\}$ is a competitive equilibrium. From the solutions obtained above, $\pi^{*}=\left(\frac{1}{\beta}-r\right) k_{0}$ so the budget constraint is satisfied as it becomes $\frac{k_{0}}{\beta} \leq\left(\frac{1}{\beta}-r\right) k_{0}+r k_{0}=\frac{k_{0}}{\beta}$. We show that $\left\{\mathbf{c}^{*}, \mathbf{l}^{*}\right\}$ is solution of the consumer's problem.

Let us consider

$$
\begin{aligned}
\Delta_{T} & =\sum_{t=0}^{T} \beta^{t}\left(c_{t}^{*}+m l_{t}^{*}\right)-\sum_{t=0}^{T} \beta^{t}\left(c_{t}+m l_{t}\right) \\
& =\sum_{t=0}^{T} \beta^{t}\left[\frac{1}{\beta}\left(k_{t}^{*}-k_{t}\right)-\left(k_{t+1}^{*}-k_{t+1}\right)+m\left(1-l_{t}\right)\right] \\
& \geq \sum_{t=0}^{T}\left[\beta^{t-1}\left(k_{t}^{*}-k_{t}\right)-\beta^{t}\left(k_{t+1}^{*}-k_{t+1}\right)\right] \\
& =-\beta^{T}\left(k_{T+1}^{*}-k_{T+1}\right)=\beta^{T} k_{T+1} \geq 0
\end{aligned}
$$

${ }^{13}$ From the first order conditions, we may have another solution

$$
\begin{aligned}
\lambda_{t}^{1} & =\beta^{t}, \\
\lambda_{t}^{2} & =\lambda_{t}^{3}=\lambda_{t}^{4}=\lambda_{t}^{5}=0, \\
k_{t} & =\frac{1}{\beta} k_{t-1}=\left(\frac{1}{\beta}\right)^{t} k_{0} . \\
c_{t} & =0, \forall t \geq 0 \\
L_{t} & =0, \forall t \geq 0 \\
l_{t} & =1, \forall t \geq 0 .
\end{aligned}
$$

Clearly it is not a solution to the planning problem as $\left(c_{t}^{*}, k_{t}^{*}, L_{t}^{*}, l_{t}^{*}\right)$ gives higher utility. In this solution, $\lim _{t \rightarrow \infty} k_{t}=\infty$ which violates boundedness of the capital stock (Assumption F2). 
As $\Delta_{T} \geq 0$ for any $T \geq 0, \sum_{t=0}^{\infty} \beta^{t}\left(c_{t}^{*}+m l_{t}^{*}\right) \geq \sum_{t=0}^{\infty} \beta^{t}\left(c_{t}+m l_{t}\right)$. This implies $\left(c_{t}^{*}, k_{t}^{*}, L_{t}^{*}, l_{t}^{*}\right)$ solves the max planning problem.

Similarly, it is easy to check that

$$
\begin{aligned}
\Delta_{T}= & \sum_{t=0}^{T} p_{t}^{*}\left[f\left(k_{t}^{*}, L_{t}^{*}\right)-k_{t+1}^{*}\right]-\sum_{t=0}^{T} w_{t}^{*} L_{t}^{*}-r k_{0} \\
& \quad-\left[\sum_{t=0}^{T} p_{t}^{*}\left[f\left(k_{t}, L_{t}\right)-k_{t+1}\right]-\sum_{t=0}^{i n f t y} w_{t}^{*} L_{t}-r k_{0}\right] \\
\geq & \beta^{T} k_{T+1} \geq 0 \\
\Rightarrow & \left.\pi^{*} \geq \sum_{t=0}^{\infty} p_{t}^{*} f\left(k_{t}, L_{t}\right)-k_{t+1}\right]-\sum_{t=0}^{T} w_{t}^{*} L_{t}-r k_{0}
\end{aligned}
$$

Therefore $\left\{\mathbf{k}^{*}, \mathbf{L}^{*}\right\}$ is a solution to the firm's problem. The markets clearing conditions are also satisfied.

\section{Discussion and Conclusion}

This paper studies existence of equilibrium in the optimal growth model with elastic labor supply. This model is the workhorse of dynamic general equilibrium theory for both endogenous and real business cycles. The results on existence of equilibrium have assumed strong conditions which are violated in some specifications of applied models.

This paper uses a separation argument to obtain Lagrange multipliers which lie in $\ell_{+}^{1}$. As the separation argument relies on convexity, strict convexity can be relaxed; this also means that assumptions on cross partials of utility functions are not needed (as in Aiyagari, et al. (1992), Coleman (1997), Datta, et al. (2002), Greenwood and Huffman (1995) and Le Van, et al. (2007)); and homogeneity of production is not needed. These above papers assume normality of leisure (rule out backward bending labor supply curves) to show that the capital path is monotonic but this is inessential to show existence of a competitive equilibrium. The representation theorem involves assumptions on asymptotic properties of the constraint set (which are weaker than Mackey continuity (see Bewley (1972) and Dechert (1982)). The assumptions ensure that the either the optimal sequence $\left\{c_{t}, l_{t}\right\}_{t=0}^{\infty}$ is either always strictly interior or always equal to zero. Thus, one does not have to impose strong conditions, either Inada conditions (see for example, Goenka, et

al. 2011), or $\lim _{\epsilon \rightarrow 0} \frac{u(\epsilon, \epsilon)}{\epsilon} \rightarrow+\infty$ as in Le Van and Vailakis (2004) to ensure that the 
sequence of labor is strictly interior. This latter condition is not satisfied, for example, in homogeneous period one utility functions. Assumptions that require compactness of the set of feasible allocations, such as $f^{\prime}(0)=\infty$ as in Aliprantis, et al. (1997) are not needed. The existence result also does not employ any differentiability assumptions. Thus, it covers both Leontief utility and production functions $Y=\min (K / v, L / u)$ and $Y / L=(1 / v) K / L$. This implies that the intensive production function, $y=f(k)$ where $y=Y / L$ and $k=K / L$ is effectively a straight line with slope $1 / v$ up to the capitallabor ratio $k^{*}=K^{*} / L^{*}$ and is horizontal thereafter. Another well known model where differentiability is violated is the Intensive Activity Analysis Production Function but existence follows from our results.

\section{Appendix}

\section{Proof of Proposition 1}

Denote $\mathbf{x}=\{\mathbf{c}, \mathbf{k}, \mathbf{l}\}$ and $\mathcal{F}(\mathbf{x})=-\sum_{t=0}^{\infty} \beta^{t} u\left(c_{t}, l_{t}\right), \Phi_{t}^{1}(\mathbf{x})=c_{t}+k_{t+1}-f\left(k_{t}, 1-l_{t}\right), \Phi_{t}^{2}(\mathbf{x})=$ $-c_{t}, \Phi_{t}^{3}(\mathbf{x})=-k_{t}, \Phi_{t}^{4}(\mathbf{x})=-l_{t}, \Phi_{t}^{5}(\mathbf{x})=l_{t}-1, \forall t, \Phi_{t}=\left(\Phi_{t}^{1}, \Phi_{t}^{2}, \Phi_{t+1}^{3}, \Phi_{t}^{4}, \Phi_{t}^{5}\right), \forall t$. The planning problem can be written as:

$$
\min \mathcal{F}(\mathbf{x}) \quad \text { s.t. } \Phi(\mathbf{x}) \leq \mathbf{0}, \mathbf{x} \in \ell_{+}^{\infty} \times \ell_{+}^{\infty} \times \ell_{+}^{\infty}
$$

where $\mathcal{F}: \quad \ell_{+}^{\infty} \times \ell_{+}^{\infty} \times \ell_{+}^{\infty} \rightarrow \mathbb{R} \cup\{+\infty\}$

$$
\Phi=\left(\Phi_{t}\right)_{t=0, \ldots, \infty}: \ell_{+}^{\infty} \times \ell_{+}^{\infty} \times \ell_{+}^{\infty} \times \ell_{+}^{\infty} \times \ell_{+}^{\infty} \rightarrow \ell_{+}^{\infty} \times \ell_{+}^{\infty} \times \ell_{+}^{\infty} \times \ell_{+}^{\infty} \times \ell_{+}^{\infty}
$$

$$
\text { Let } \begin{aligned}
C & =\operatorname{dom}(\mathcal{F})=\left\{\mathbf{x} \in \ell_{+}^{\infty} \times \ell_{+}^{\infty} \times \ell_{+}^{\infty} \mid \mathcal{F}(\mathbf{x})<+\infty\right\} \\
\Gamma & =\operatorname{dom}(\Phi)=\left\{\mathbf{x} \in \ell_{+}^{\infty} \times \ell_{+}^{\infty} \times \ell_{+}^{\infty} \mid \Phi_{t}(\mathbf{x})<+\infty, \forall t\right\} .
\end{aligned}
$$

The following Lemma is an extension of Le Van and Saglam (2004) to the case of the optimal growth model with endogenous labor-leisure choice.

Lemma 1. Let $\mathbf{x}, \mathbf{y} \in \ell_{+}^{\infty} \times \ell_{+}^{\infty} \times \ell_{+}^{\infty}, T \in \mathbb{N}$. Define

$$
x_{t}^{T}(\mathbf{x}, \mathbf{y})=\left\{\begin{array}{l}
x_{t} \text { if } t \leq T \\
y_{t} \text { if } t>T
\end{array} .\right.
$$

Suppose that the two following assumptions are satisfied: 
T1: If $\mathbf{x} \in C, \mathbf{y} \in \ell_{+}^{\infty} \times \ell_{+}^{\infty} \times \ell_{+}^{\infty}$ satisfy $\forall T \geq T_{0}, \mathbf{x}^{T}(\mathbf{x}, \mathbf{y}) \in C$, then $\mathcal{F}\left(\mathbf{x}^{T}(\mathbf{x}, \mathbf{y})\right) \rightarrow \mathcal{F}(\mathbf{x})$ when $T \rightarrow \infty$.

T2: If $\mathbf{x} \in \Gamma, \mathbf{y} \in \Gamma$ and $\mathbf{x}^{T}(\mathbf{x}, \mathbf{y}) \in \Gamma, \forall T \geq T_{0}$, then

a) $\Phi_{t}\left(\mathbf{x}^{T}(\mathbf{x}, \mathbf{y})\right) \rightarrow \Phi_{t}(\mathbf{x})$ as $T \rightarrow \infty$

b) $\exists M$ s.t. $\forall T \geq T_{0},\left\|\Phi_{t}\left(\mathbf{x}^{T}(\mathbf{x}, \mathbf{y})\right)\right\| \leq M$

c) $\forall N \geq T_{0}, \lim _{t \rightarrow \infty}\left[\Phi_{t}\left(\mathbf{x}^{T}(\mathbf{x}, \mathbf{y})\right)-\Phi_{t}(\mathbf{y})\right]=0$.

Let $\mathbf{x}^{*}$ be a solution to $(P)$ and $\mathbf{x}^{0} \in C$ satisfies the Slater condition:

$$
\sup _{t} \Phi_{t}\left(\mathbf{x}^{0}\right)<0
$$

Suppose $\mathbf{x}^{T}\left(\mathbf{x}^{*}, \mathbf{x}^{0}\right) \in C \cap \Gamma$. Then, there exists $\boldsymbol{\Lambda} \in \ell_{+}^{1} \backslash\{0\}$ such that

$$
\mathcal{F}(\mathbf{x})+\Lambda \Phi(\mathbf{x}) \geq \mathcal{F}\left(\mathbf{x}^{*}\right)+\Lambda \Phi\left(\mathbf{x}^{*}\right), \forall \mathbf{x} \in(C \cap \Gamma)
$$

and $\Lambda \Phi\left(\mathbf{x}^{*}\right)=0$

Proof Lemma: It is easy to see that $\ell_{+}^{\infty} \times \ell_{+}^{\infty} \times \ell_{+}^{\infty}$ is isomorphic with $\ell_{+}^{\infty}$, since, for example, there exists an isomorphism

$$
\begin{gathered}
\Pi: \ell_{+}^{\infty} \rightarrow \ell_{+}^{\infty} \times \ell_{+}^{\infty} \times \ell_{+}^{\infty} \\
\Pi(\mathbf{x})=\left(\left(x_{0}, x_{3}, x_{6}, \ldots\right)\left(x_{1}, x_{4}, x_{7}, \ldots\right),\left(x_{2}, x_{5}, x_{8}, \ldots\right)\right)
\end{gathered}
$$

and

$$
\Pi^{-1}(\mathbf{u}, \mathbf{v}, \mathbf{s})=\left(u_{0}, v_{0}, s_{0}, u_{1}, v_{1}, s_{1}, u_{2}, v_{2}, s_{2}, \ldots\right)
$$

Thus, there exists an isomorphism $\Pi^{\prime}:\left(\ell_{+}^{\infty} \times \ell_{+}^{\infty} \times \ell_{+}^{\infty}\right)^{\prime} \rightarrow\left(\ell_{+}^{\infty}\right)^{\prime}$. It follows from Theorem 1 in Le Van and Saglam (2004) that there exists $\bar{\Lambda} \in\left(\ell_{+}^{\infty} \times \ell_{+}^{\infty} \times \ell_{+}^{\infty}\right)^{\prime}$. Let $\Lambda=\Pi^{\prime}(\bar{\Lambda}) \in\left(\ell_{+}^{\infty}\right)^{\prime}$. Then, the results are derived by the analogous arguments where a standard separation theorem used ${ }^{14}$ as in the Theorem 2 in Le Van and Saglam (2004). Note that T1 holds when $F$ is continuous in the product topology. T2c is satisfied if there is asymptotically insensitivity, i.e. if $x$ is changed only on a finitely many values the constraint value for large $t$ does not change that much (Dechert 1982). T2c is the asymptotically non-anticipatory assumption and requires $\Phi^{i},(i=1, . ., 5)$, to be weak-* continuous (Dechert 1982). T2b holds when $\operatorname{dom}\left(\Phi^{i}\right)=\ell^{\infty}$ and $\Phi^{i}$ is continuous (see

\footnotetext{
${ }^{14}$ As the Remark 6.1.1 in Le Van and Dana (2003), assumption $f_{k}(0,1)>1$ is equivalent to the Adequacy Assumption in Bewley (1972) and this assumption is crucial to have equilibrium prices in $\ell_{+}^{1}$ since it implies that the production set has an interior point. Subsequently, it allows using a separation theorem in the infinite dimensional space to obtain Lagrange multipliers.
} 
Le Van and Saglam (2004)). As each $\Phi^{i}$ is continuous, $\Phi$ is continuous in the product topology. ${ }^{15}$

Now, we are in a position to prove Proposition 1. We first check that the Slater condition holds. Indeed, since $f_{k}^{\prime}(0,1)>1$, then for all $k_{0}>0$, there exists some $0<\widehat{k}<k_{0}$ such that: $0<\widehat{k}<f(\widehat{k}, 1)$ and $0<\widehat{k}<f\left(k_{0}, 1\right)$. Thus, there exists two small positive numbers $\varepsilon, \varepsilon_{1}$ such that:

$$
0<\widehat{k}+\varepsilon<f\left(\widehat{k}, 1-\varepsilon_{1}\right) \text { and } 0<\widehat{k}+\varepsilon<f\left(k_{0}, 1-\varepsilon_{1}\right) .
$$

Denote $\mathbf{x}^{0}=\left(\mathbf{c}^{0}, \mathbf{k}^{0}, \mathbf{l}^{0}\right)$ such that $\mathbf{c}^{0}=(\varepsilon, \varepsilon, \ldots), \mathbf{k}^{0}=\left(k_{0}, \widehat{k}, \widehat{k}, \ldots\right), \mathbf{l}^{0}=\left(\varepsilon_{1}, \varepsilon_{1}, \ldots\right)$. We have

$$
\begin{aligned}
& \Phi_{0}^{1}\left(\mathbf{x}^{0}\right)=c_{0}+k_{1}-f\left(k_{0}, 1-l_{0}\right) \\
&=\varepsilon+\widehat{k}-f\left(k_{0}, 1-\varepsilon_{1}\right)<0 \\
& \Phi_{1}^{1}\left(\mathbf{x}^{0}\right)=c_{1}+k_{2}-f\left(k_{1}, 1-l_{1}\right) \\
&=\varepsilon+\widehat{k}-f\left(\widehat{k}, 1-\varepsilon_{1}\right)<0 \\
& \Phi_{t}^{1}\left(\mathbf{x}^{0}\right)=\varepsilon+\widehat{k}-f\left(\widehat{k}, 1-\varepsilon_{1}\right)<0, \forall t \geq 2 \\
& \Phi_{t}^{2}\left(\mathbf{x}^{0}\right)=-\varepsilon<0, \forall t \geq 0, \quad \Phi_{0}^{3}\left(\mathbf{x}^{0}\right)=-k_{0}<0 \\
& \Phi_{t}^{3}\left(\mathbf{x}^{0}\right)=-\widehat{k}<0, \quad \forall t \geq 1, \quad \Phi_{t}^{4}\left(\mathbf{x}^{0}\right)=-\varepsilon_{1}<0, \quad \forall t \geq 0 \\
& \Phi_{t}^{5}\left(\mathbf{x}^{0}\right)=\varepsilon_{1}-1<0, \forall t \geq 0 .
\end{aligned}
$$

Therefore, the Slater condition is satisfied. Now, it is obvious that, $\forall T, \mathbf{x}^{T}\left(\mathbf{x}^{*}, \mathbf{x}^{0}\right)$ belongs to $\ell_{+}^{\infty} \times \ell_{+}^{\infty} \times \ell_{+}^{\infty}$. As in Le Van and Saglam (2004), Assumption T2 of Lemma1 is satisfied. We now check Assumption T1. For any $\widetilde{\mathbf{x}} \in C, \widetilde{\widetilde{\mathbf{x}}} \in \ell_{+}^{\infty} \times \ell_{+}^{\infty} \times \ell_{+}^{\infty}$ such that for any $T$, $\mathbf{x}^{T}(\widetilde{\mathbf{x}}, \widetilde{\mathbf{x}}) \in C$ we have

$$
\mathcal{F}\left(\mathbf{x}^{T}(\widetilde{\mathbf{x}}, \widetilde{\widetilde{\mathbf{x}}})\right)=-\sum_{t=0}^{T} \beta^{t} u\left(\widetilde{c_{t}}, \widetilde{l_{t}}\right)-\sum_{t=T+1}^{\infty} \beta^{t} u\left(\widetilde{\widetilde{c_{t}}}, \widetilde{l_{t}}\right) .
$$

As $\widetilde{\widetilde{\mathbf{x}}} \in \ell_{+}^{\infty} \times \ell_{+}^{\infty} \times \ell_{+}^{\infty}, \sup _{t}\left|\widetilde{\widetilde{c}}_{t}\right|<+\infty$, there exists $m>0, \forall t,|\widetilde{\widetilde{c}}| \leq m$. Since $\beta \in(0,1)$ we have

$$
\sum_{t=T+1}^{\infty} \beta^{t} u(m, 1)=u(m, 1) \sum_{t=T+1}^{\infty} \beta^{t} \rightarrow 0 \text { as } T \rightarrow \infty .
$$

Hence, $\mathcal{F}\left(\mathbf{x}^{T}(\widetilde{\mathbf{x}}, \widetilde{\mathbf{x}})\right) \rightarrow \mathcal{F}(\widetilde{\mathbf{x}})$ when $T \rightarrow \infty$. Taking account of the Lemma, we get (1)-(6). Finally, we obtain (7)-(9) from the Kuhn-Tucker first-order conditions.

\footnotetext{
${ }^{15}$ Indeed, if $V=\Pi_{i=1}^{5} V^{i}$ is basic open in $\ell_{+}^{\infty} \times \ell_{+}^{\infty} \times \ell_{+}^{\infty} \times \ell_{+}^{\infty} \times \ell_{+}^{\infty}$ then $\Phi^{-1}(V)=\cap_{i=1}^{5}\left(\Phi^{i}\right)^{-1}\left(V^{i}\right)$ is open.
} 


\section{References}

[1] Aiyagari, S.R., Christiano, L.J. and Eichenbaum, M. (1992) The output, employment, and interest rate effects of government consumption, J. Mon. Econ. 30, 73-86

[2] Aliprantis, C.D., D.J. Brown and O. Burkinshaw (1997) New proof of the existence of equilibrium in a single sector growth model, Macro. Dynamics 1, 669-679.

[3] Bewley, T.F. (1972) Existence of equilibria in economies with finitely many commodities, J. Econ. Theory 4, 514-540.

[4] Bewley T. F. (1982) An integration of equilibrium theory and turpike theory, $J$. Math. Econ. 10, 233-267.

[5] Coleman II , W.J. (1997) Equilibria in distorted infinite-horizon economies subject to taxes and externalities, J. Econ. Theory 72, 446-461.

[6] Dana, Rose-Anne and C. Le Van (1991) Equilibria of a stationary economy with recursive preferences, Journal of Optimization Theory and Applications 71, 289-313.

[7] Datta, M., L.J. Mirman and K.L. Reffet (2002) Existence and uniqueness of equilibrium in distorted economies with capital and labor, J. Econ. Theory 103, 377-410.

[8] Dechert, W.D. (1982) Lagrange multipliers in infinite horizon discrete time optimal control models, J. Math. Econ. 9, 285-302.

[9] Goenka A, C. Le Van and M-H. Nguyen (2012) Existence of competitive equilibrium in an optimal growth model with heterogeneous agents and endogeneous leisure, Macroeconomic Dynamics,16 (S1), 33-51.

[10] Greenwood, J. and G. Huffman (1995) On the existence of nonoptimal equilibria in dynamic stochastic economies, J. Econ. Theory 65, 611-623.

[11] Hansen, G.D. (1985) Indivisible labor and the business cycle, J. Mon. Econ. 16, 309-327.

[12] Iwasa, K. and G. Sorger (2018) Periodic solutions of the one-sector growth model: The role of income effects, J. Math. Econ. 78, 59-63.

[13] Kamihigashi, T. (2015) Multiple interior steady states in the Ramsey model with elastic labor supply, Int. J. Econ. Theory 11, 25-37.

[14] Le Van, C. and R.A. Dana (2003), Dynamic Programming in Economics. Dordrecht: Kluwer Academic Publishers. 
[15] Le Van C. and H. Saglam (2004) Optimal growth models and the Lagrange multiplier, J. Math. Econ. 40, 393-410.

[16] Le Van, C and M.H. Nguyen and Y. Vailakis (2007) Equilibrium dynamics in an aggregative model of capical accumulation with heterogeneous agents and elastic labor, J. Math. Econ. 43, 287-317.

[17] Le Van, C. and Y. Vailakis (2003) Existence of a competitive equilibrium in a one sector growth model with heterogeneous agents and irreversible Investment, Econ. Theory 22, 743-771.

[18] Le Van, C and Y. Vailakis (2004) Existence of competitive equilibrium in a singlesector growth model with elastic labor, Cahiers de la MSE, N0 2004-123.

[19] Peleg B. and M. E. Yaari, (1970) Markets with countably many commodities, Int. Econ. Rev. 11, 369-377.

[20] Rogerson, R. (1988) Indivisible labor, lotteries and equilibrium, J. Mon. Econ. 21, 3-16.

[21] Sorger, G. (2018) Cycles and chaos in the one-sector growth model with elastic labor supply. Econ. Theory 65, 55-77.

[22] Yano, M. (1984) Competitive equilibria on turnpikes in a McKenzie economy I: A neigbhorhood turnpike theorem, Int. Econ. Rev. 25(3), 695-717.

[23] Yano, M. (1990) Von Neumann facets and the dynamic stability of perfect foresight equilibrium paths in neo-classical trade models, J. Econ. 51(1), 27-69.

[24] Yano, M. (1998) On the dual stability of a von Neumann facet and the inefficacy of temporary fiscal policy, Ecta., 66(2), 427-451. 